\title{
BMJ Open Exploration of rural physicians' lived experience of practising outside their usual scope of practice to provide access to essential medical care (clinical courage): an international phenomenological study
}

\author{
Jill Konkin, ${ }^{1}$ Laura Grave, ${ }^{2}$ Ella Cockburn, ${ }^{2}$ Ian Couper, ${ }^{3}$ Ruth Alison Stewart, ${ }^{4}$ \\ David Campbell, ${ }^{5}$ Lucie Walters (1) ${ }^{6}$
}

To cite: Konkin J, Grave L Cockburn E, et al. Exploration of rural physicians' lived experience of practising outside their usual scope of practice to provide access to essential medical care (clinical courage): an international phenomenological study. BMJ Open 2020;10:e037705. doi:10.1136/ bmjopen-2020-037705

- Prepublication history for this paper is available online. To view these files, please visit the journal online (http://dx.doi org/10.1136/bmjopen-2020037705).

Received 13 February 2020 Revised 17 April 2020 Accepted 21 July 2020

Check for updates

(c) Author(s) (or their employer(s)) 2020. Re-use permitted under CC BY-NC. No commercial re-use. See rights and permissions. Published by BMJ.

For numbered affiliations see end of article.

Correspondence to

Dr Lucie Walters;

lucie.walters@adelaide.edu.au

\section{ABSTRACT}

Objectives Rural doctors describe consistent pressure to provide extended care beyond the limits of their formal training in order to meet the needs of the patients and communities they serve. This study explored the lived experience of rural doctors when they practise outside their usual scope of practice to provide medical care for people who would otherwise not have access to essential clinical services.

Design A hermeneutic phenomenological study. Setting An international rural medicine conference. Participants All doctors attending the conference who practised medicine in rural/remote areas in a predominantly English-speaking community were eligible to participate; 27 doctors were recruited.

Interventions Semi-structured interviews were conducted. The transcripts were initially read and analysed by individual researchers before they were read aloud to the group to explore meanings more fully. Two researchers then reviewed the transcripts to develop the results section which was then rechecked by the broader group.

Primary outcome measure An understanding of the lived experiences of clinical courage.

Results Participants provided in-depth descriptions of experiences we have termed clinical courage. This phenomenon included the following features: Standing up to serve anybody and everybody in the community; Accepting uncertainty and persistently seeking to prepare; Deliberately understanding and marshalling resources in the context; Humbly seeking to know one's own limits; Clearing the cognitive hurdle when something needs to be done for your patient; Collegial support to stand up again. Conclusion This study elucidated six features of the phenomenon of clinical courage through the narratives of the lived experience of rural generalist doctors.

\section{BACKGROUND}

Normative values in clinical medicine tend to frame quality and safety assuming proximal and timely access for patients to specialist and
Strengths and limitations of this study

- Collectively, the researchers have rural clinical practice experience across three continents, enabling interpretation of interviews as informed insiders.

- All participants in the study attended the same international rural health conference which may have resulted in a sample with strong engagement in rural health issues.

- The participants in this study practised rural medicine in a broad range of countries adding to the international transferability of the results.

subspecialist care. ${ }^{1}$ Rural doctors describe consistent pressure to provide extended care beyond the limits of their formal training in order to meet the needs of the patients and communities they serve. Factors creating this tension include poorer patient access to care, healthcare professional undersupply, hospital resource limitations, overlapping clinical roles and training constraints. ${ }^{2}$ In an Australian-wide study of 465 medical students at the completion of their full academic year rural clinical placements, $24 \%$ reported that they felt rural practice was too hard. ${ }^{3}$

In remote and rural areas, distance provides a significant barrier to patient access to specialist care, and at times rural doctors can be faced with the choice of providing a service to patients which is not comfortably within their own scope, or facing the reality that their patient or their community will go without. Past President of Australian College of Rural and Remote Medicine, John (Jack) Shepherd is credited with coining the term clinical courage, defined by John Wootton as "that space where the needs of our patients 
and the extent of our training and experience intersect". ${ }^{45}$ To date, reference to clinical courage is uncommon, with minimal academic exploration of this phenomenon. ${ }^{46}$

This research study sought to understand the lived experiences of rural doctors at times when they have found themselves working at the edges of their scope of practice to provide essential medical care.

\section{METHODS}

Hermeneutic phenomenology was used as the study design. Phenomenology seeks to explore the lived experiences of rural doctors providing care for patients outside of their comfort zone in order to describe the 'essence' of this state. ${ }^{7}$ Hermeneutics recognises the study participants' self-interpretation of experiences in the process of meaning making. In contrast to empiricist phenomenology, which purports the need to bracket the researchers' presuppositions of clinical courage ${ }^{8}$ hermeneutic phenomenology gives credence to researchers sharing their experience with the participants in the co-construction of the meaning in interviews. ${ }^{9}$ The study used an interpretive constructivist lens assuming no single reality exists, but rather that individuals create social constructs through the interpretation of their own experiences. ${ }^{7}$ The research team sought to co-construct understandings of clinical courage as features emerged from the material. In this project, DC, IC, JK, RAS and LW are experienced rural doctors, while EC and LG are Australian junior doctors who previously undertook a longitudinal integrated placement for a full academic year in a rural setting. ${ }^{10}$

Participant recruitment occurred at the WONCA Rural Conference, 14th World Rural Health Conference in Cairns, Australia. Rural doctors who practised medicine in a predominantly English-speaking community anywhere in the world were invited to participate through fliers placed on seats in the conference venue. Consent was sought if they approached the booth where LG and EC were in attendance. Snowball sampling occurred by asking participants to invite colleagues who could add diversity to the cohort across a range of demographics including gender, self-reported stage of career, and remoteness of clinical practice.

Semi-structured face-to-face interviews were undertaken using the interview guide provided in box 1 . Interviews were 30 to $60 \mathrm{~min}$ duration. Recordings were de-identified and allocated a random number. They were then anonymised and transcribed by a transcription service with which the research team holds a confidentiality agreement. Transcriptions were returned to participants to review and approve prior to analysis.

Each transcript was read and annotated for holistic meanings by at least two members of the research team. The international research team then met via videoconference and read a selection of interviews aloud as a group to create consensus regarding the emerging features and discuss the overarching meanings. Notes were taken at

\section{Box 1 Semi-structured interview questions}

\section{Interview questions}

1. Tell me a bit about yourself and your current rural practice.

2. Please describe your lived experience of pushing the boundaries of your own scope of practice for your patients.

3. What contexts or situations have typically influenced or affected your experiences?

4. What would you call this?

5. Can you describe a recent time when you have had to draw on your own clinical courage or where you have witnessed a rural colleague draw on their clinical courage?

6. Can you describe your own experience of clinical courage?

7. In this study, we are hoping to develop a better understanding of country doctor's experiences of managing the challenges they face in their clinical roles. Do you have anything else to add before we conclude the interview?

these meetings and added to NVivo along with annotations on the transcripts. All interviews were read aloud together by LW and JK and quotes were chosen to demonstrate congruence across the interviews as well as seeking to illustrate the breadth of the experiences.

\section{Participant and public involvement}

The initial study plan was developed through an international rural research consultation workshop at the Norwegian Centre for Rural Medicine in Tromso, Norway in 2016, where around 30 rural doctors and rural health researchers came together to explore research opportunities considered important to progress the rural health agenda. Snowball sampling engaged participants in choosing others to contribute to the study. Participants were told the study would explore the intersection of access and quality in rural medicine in order to allow the first part of the interview to explore the phenomenon without naming it clinical courage (see interview guide in box 1). Several presentations at rural conferences enabled authors to draw on reactions of rural doctors to reconsider our interpretations of initial results.

\section{RESULTS}

In total, 27 interviews were performed, with participants ranging across the stage of their careers and a range of countries including Australia (15 participants), Canada (5), USA (3), New Zealand (2), South Africa (2) and one each from Scotland and Papua New Guinea. Several participants had worked in more than one country during their career. Early career participants included two doctors in their first year after medical school, and three doctors 5-7 years post-graduation (table 1). Middle career participants all described being greater than 7 years post fellowship, and participants who described themselves as experienced clinicians had greater than 20 years of clinical experience often across a number of remote and rural sites. 
Table 1 Demographics of research participants

\begin{tabular}{llll}
\hline Self-reported stage of career & Female & Male & Participant numbers \\
\hline Early & Two & Three & $15,18,19,20,25$ \\
Middle & Seven & Four & $3,5,11,12,13,14,17,22,23,24,26$ \\
Experienced & Nine & Two & $1,2,4,6,7,8,9,10,16,21,27$ \\
\hline
\end{tabular}

Participants identify that the choice of rural practice requires courage.

The courage comes from making the decision to put yourself in that situation. So at the beginning of my contract, when I took up that position as senior doctor. And the same for all these guys that put themselves in remote or rural practice, they know what they're putting themselves in for. They know that there is the potential for somebody really sick to come along or for some emergency to happen that they can't fully deal with within their scope of practice and in their experience. And they know they're going to be pushed to their limits, and they know they're going to be outside of their comfort zone. [26]

They describe working outside their comfort zone with terms like courage and humility and doing one's best for the patients.

I think the important thing is that doctors have to acknowledge or ... understand that it's okay to be outside your comfort zone sometimes. ... If you rely on your training and your ingenuity and the support you have, it makes it easier. [7]

You know it's not about confidence, it's about clinical courage and there has to be humility there, like they're just really important, because the fates can deal you anything they want, and you can't go into it thinking you can do everything. Like, you just have to be recognising that I'm going to be okay, I'll give it a shot. I'm a little nervous, but I'll give it a shot and do whatever I need to do for the patient, which is what it's all about, it's all about the patient. It's not about me. [21]

I think of courage more as the willingness to go where there's a risk, willingness to try even if I fail because I have no choice; it's me or nobody. So I don't know if that's courage. Some would call it foolhardiness, some would call it head in the sand. I think it's just accepting the reality of where you are and doing the best you can and being okay with however it turns out, recognising that you gave it your best. So yes it's courageous but it's also born out of practise over years. You become more courageous, you become more willing to put yourself there because you've seen it work out well in the past and so it gives you courage to even do more. [1]

Rural doctors do what they do through a deep connection to community and that connection goes both ways.
It's part of the reason you go into rural health-is that you want to be intimately involved with the community. [3]

You step up to the plate to help the community because it's the right thing to do, and the people in the community are your community. [21]

I think because of the nature of our interactionswell definitely for me, the nature of my interactions means that I get to know the patients very well; I get to know the community well and therefore I feel a sense of responsibility, maybe a sense of duty; but I think definitely a sense that I will be supported when I make decisions that are in the best interests of that patient. So I think about the times where adverse outcomes have happened in a remote setting and I haven't been ostracised. [1]

I think once people understand their patients and their community, I think people build courage. [3]

Rural doctors are attracted to the broad scope of rural practice even though it can be daunting.

All the things you learn about rural doctors, they're adventurous and they have attention deficit disorder and this and this and that, I think it's all true for me, and I just like doing the full job .... So it's a lot of fun. There's a lot of terror along the way too. [7]

Working at the edge of comfort is part of practice for rural doctors. Some participants identify that a practice confined to one's comfort zone leads to atrophy of knowledge and skills as well as confidence.

And I would tell students who would come to visit and do a month with me or whatever, ... that I better [know] my capabilities because every day I spent my time at the margin of my capabilities, I was always at the margin of my competence. So I often would slip over and back and I always knew where that edge was whereas many of my colleagues practising in the city practised in their comfort zone. And over time their scope of practice became more and more restricted, they became less and less confident and less and less courageous about doing anything that was anywhere close to the margin of their skills whereas I was stretched every day. And it was a great life, good and bad. [1]

Participants identified that they are comfortable with uncertainty. 
I've come to accept the fact, and not resigned to the fact, but accept the fact that what I do involves uncertainty, and I'm always being pushed beyond my, not definitely comfort zone, but maybe skillset and knowledge zone ... you have to have sometimes courage with every patient, because you can't possibly know and learn everything ... [27]

Delivering safe, quality healthcare to patients when stretched beyond ones comfort is top of mind for rural doctors. They describe that this requires clear thinking, having a systematic approach to patients, and includes reflection on their own motivation for going beyond their comfort zone.

We're very well trained .... And as long as they don't panic and they approach things in a systematic manner, to do the things we're trained to do, the ABCs, and to do an appropriate assessment before you jump into action and set your priorities I think then you're prepared for anything that happens, regardless of the severity, you have an approach to meet there. [2]

So I think in my mind I think clinical courage sort of takes you to a point and then you may be beyond that point, then you, you know, it may be dangerous. I don't want to ever be in that situation where I can reflect back and think that I've been dangerous in terms of the care of my patients. [4]

There's a lot of churning of emotions and thought processes; am I doing this because I just want to be a hero, is this really in the patient's best interests? [22]

There is an element of clearing a mental hurdle that allows rural doctors to move beyond their comfort zone. It is for the patient and their doctors to determine who is the best person to do what needs to be done. Having colleagues to confer with is helpful.

Clinical courage is crossing the cognitive threshold. Because quite often when you come to that you know what you need to do but because you don't do it all the time ... you know you want to do it .... [O]nce you start that you can't step backwards .... But making the decision to do that, once the decision is made ... it's about calling it and saying 'yep, this is what we have to do' and then once you vocalise that then it makes it a bit easier. [3]

So sometimes you have to have the courage of your conviction and I guess that's where they talk about clinical courage. You have to say, 'I have never done a chest tube on a baby, but a chest tube is a chest tube is a chest tube, it's just smaller,' and maybe you have to be a bit more careful. And maybe you [the doctor] will get coronary vasospasm, but ... you just have to sort of say, 'If I don't do something things will definitely get worse. If I do something there's a $50 \%$ chance or more that the patient will get better.' [7]

I think in my own case you draw upon experience, you draw upon the fact that something needs to be done. Often when I get totally petrified in a situation, I used to be a big rock climber, I think of being in a difficult situation on the rock and you just have to do something. You either have to go up or you have to go down, or you're going to fall off, so you have to make a move. And there's a saying, it's better to make the wrong decision than to make no decision at all. So sometimes you just have to say to yourself, 'I think this is what we have to do,' and usually in my case most of my life there's always been another doctor or two around, who are supportive and at least add numbers if not experience necessarily, and that helps. [7] I spoke to a specialist anaesthetist who is based in [town name], which is another remote area, and he was really useful because he basically called the situation as it was .... And that was a really steadying influence. If I hadn't had him, I think I really would have struggled, because it just brought back my focus to what I had to do. [12]

Rural doctors acknowledge that the circumstances of rural practice require that they act. When they act, they identify that they need to stay focused no matter how anxious or scared they feel.

Because it's my job and I'm the most qualified one there. And what's the option? There's no option. And I put my hand up to be there, so I have to do it. [6]

... knowing that that can happen and that you're going to have to deal with it and the consequences as and when they arise; from an emotional level, from a professional level, you know, from an impact on your community and individual. You're putting your hand up and saying, 'I'll stand there. I'll do that.' That's where the courage comes. [26]

So, one of the things that-one of the most important clinical interventions to do with someone-I can't remember who taught me this, but is when you walk into a room where everybody's glued to the walls, just take your own pulse, make sure you're okay, and then just breathe out. And, even if you've got a really difficult situation, it's your job to stay calm and figure out what to do next. And, if you do that you'll be okay. And, you may be in the middle of an impossible situation, but that doesn't mean you need to get out of control, you just have to work through it. [21]

It's not a lack of human emotion but it's trying to remove yourself from how scary it really is and trying to focus-task focus on what you need. So, the combination of those skills that you know will get you through, but trying to-knowing that if you think about what could happen if you get it wrong, to try not to do that and really focus on, 'This is what I need to do here and now.' [5]

And sometimes you have to have the courage to say "NO". 
And then they [consultants] push you to do stuff that you maybe shouldn't. So sometimes you have to say, 'No, I'm not going to do this. I'm going to send this patient down to you.' So don't get squashed or squeezed into a spot that you don't want to be. You have the right to say no if it just feels wrong to you or too dangerous to you or the patient. Some stuff is cowboy stuff and you shouldn't be doing it. [7]

They have to persist even when the outcomes aren't what was hoped for.

... there was a crash C-section with a poor outcome, the baby came out and I resuscitated the baby and I had to do everything in terms of intubation and lines to keep this baby alive but we ended up shipping the baby out and it died within the next 24 hours. So I think those situations are always tough, you know, especially when you're dealing with kids or infants and death, those are tough situations. So I'm not sure if that represents clinical courage or just the difficulties of clinical practice when you lose, especially like you say when children die or infants die that's always difficult. [4]

I think some form of resilience because if you're not a resilient practitioner you can easily withdraw and go, 'I'm not going to do this ever again because it was too hard, it was too tough.' [20]

Unfortunately, health delivery systems can be unsupportive contexts, failing to afford rural doctors with the opportunity to work at their full scope. Rural doctors have the knowledge and skills to deliver a much broader level of service but lack the resources and system support to do so.

It's not always the right thing to do in a context. I think its very context specific and so that's where the fuzziness comes in .... There's anxiety of are you good enough to do what you think you can do and bring benefit to the patient. Is this the right context to do it in and working out even what your team is telling you. I think that's very useful. But it's also important professionally to realise when you sometimes have to block off that feedback which is a very, very precarious situation and sometimes it's the wider once you've done it often as well. Of that extreme acts ... if it goes well then at least you've got something and you're probably less likely to be questioned about it at the time. I think sometimes if it goes wrong then you expose yourself to an nth degree. [13]

... with credentialing and limiting scope of practice and specialists saying well you shouldn't do that as a GP, we're actually limiting what people do and at the end of the day it's the patients that suffers. For me as a professional I'm not, I don't need to do this stuff but you're in a situation where you want to be able to help your patients in every situation. [10]
Having a supportive culture is also key to being able to practice at the edge of comfort.

I think our practice supported that and the culture in our hospital, which is built through the collective action of individuals over time. But over time a place develops a culture that's supportive, that's collegial, that's mutually respectful. That isn't something that just happens and so I would just encourage anyone who's in a place where that culture doesn't exist to keep acting in that way and get some colleagues to act in that way. And then if everyone acts in that way after a while you build a culture; it takes 10, 20 years for that to happen I think. [1]

\section{DISCUSSION}

Aristotle reportedly emphasised that courageous action required: (1) a morally worthy goal or ideal, (2) a dangerous situation and (3) consideration of potential value and threats of any action. ${ }^{11}$ This study builds on Aristotle's courageous action when describing the lived experience of doctors who chose to work outside their usual scope of practice to deliver care to their patients in remote and rural contexts. These features of clinical courage arose from the voices of the rural doctor participants of this study.

\section{Standing up to serve anybody and everybody in the community}

Participant narratives demonstrate a deep commitment to providing healthcare to rural communities. Participants describe a deliberated altruistic decision to put themselves into positions where they will feel out of their depth clinically and risk distress, professional isolation and potentially psychological trauma. This altruistic decision is often based on their sense of belonging to a community, and their drive for fair treatment for people they identify with, and for whom they are prepared to tolerate the risk associated with their actions. This study positions motivation to serve one's own rural community as a morally worthy goal which doctors committed to both when initially joining a community and recurrently when returning following difficult days. ${ }^{12}$ Responsibility for their patient care was more emotionally intense and complex due to entwined relationships with patients who were also friends or colleagues. ${ }^{13}$

\section{Accepting uncertainty and persistently seeking to prepare}

Uncertainty is an accepted component of medicine; however, this is usually described within the context of a diagnostic dilemma and the clinical reasoning processes used to manage this. ${ }^{14}$ Participant narratives in this study point to uncertainty relating to how often and to what extent their own clinical skills will be stretched. Clinical courage has been linked previously with motivation to acquire acute care skills where GP registrars in Australia "took initiative and pushed boundaries to extend their clinical 
skills" ${ }^{15}$ From this study's narratives, skills acquisition included not only acute resuscitation skills but niche skills required to appropriately manage patients with complex, less acute, problems in their home communities, such as mental health, chemotherapy and renal failure. It also identified the on-the-spot adaptive expertise of transferring skills used in different situations to a new situation as well as figuring out what to do in real time with in a critical situation. ${ }^{16} 17$

\section{Deliberately understanding and marshalling resources in the context}

The narratives identified a clear sense of not having the personnel and equipment which might be available in better resourced areas when managing patients. Familiarity with the context of practice and relationships with local team members and distance support and retrieval systems enabled rural doctors to maximise the available local resources for the benefit of their patients. There was some discussion about the concerns doctors have when working in an unfamiliar clinical context such as when doing locum jobs or when new to an area. Unfamiliarity with the context created increased anxiety as doctors were less aware of the resources they could call on in challenging clinical circumstances.

\section{Humbly seeking to know one's own limits}

The self-assessment literature cautions that overconfident self-judgements are not uncommon, and that experience can increase confidence thereby increasing the risk of overestimating one's own skills. ${ }^{18}$ The study narratives consistently describe conscientious 'intellectual humility' where doctors seek to understand the boundaries of their knowledge and skills despite the cognitive and emotional effort this requires. Humility is not a passive process. ${ }^{18}$ These doctors, working in low resource settings at a distance from tertiary care and, often, secondary care centres, do not conflate confidence with competence. In the routine testing of their limits as part of their everyday practice, they describe learning how to test their limits. Limits are sought through deliberate practice and testing, self-reflection and critical discourse with experts and peers, patients and community members. ${ }^{19}$

\section{Clearing the cognitive hurdle when something needs to be done for your patient}

Courageous action requires a difficult, painful or dangerous situation. ${ }^{11}$ Previously, in the context of caring for suicidal patients, courage has been described as a clinician doing "the very thing we feel least inclined to do" to make a difference for the patient. ${ }^{20}$ In this study, participants describe the point of action following their assessment of the benefits and risks associated with this action. At this point, when they are clear that there is no one else better able to provide that care, they must switch from a state of risk assessment and self-critique and focus on the task at hand with confidence.

\section{Collegial support to stand up again}

Participants described a persistent willingness to work at the edge of one's limits and perform beyond one's comfort zone. The benefits of supportive colleagues in facilitating and maintaining clinical courage was emphasised. Participants consistently described the value of justin-time discourse with colleagues in exploring the risks and benefits of proposed management plans, especially when colleagues had a strong familiarity with the context of their remote/rural community. In addition, peer reflection added to their own self-reflections following significant events. The importance of peer assessment being context specific is critical to enabling understanding and better supporting rural doctors to continue to choose to step up again to be clinically courageous.

\section{Limitations}

This study has some potential limitations. All participants in the study attended the same international rural health conference, which may have resulted in a sample with strong engagement in rural health issues. It is interesting to note the large number of female participants in this study in a field that is historically male dominated. Snowball sampling, contingent of female researchers and two female medical student interviewers, may have influenced the diversity of participants. There is a possibility that this limited the diversity of participants who might have viewed their clinical work differently. The conference setting provided quiet but not completely private contexts for interviews and may have limited sharing of sensitive information with the interviewers. This study begins to explore the phenomenon of clinical courage as it occurs in rural doctors; however, further studies will be required to develop a more comprehensive understanding of this important clinical phenomenon.

\section{CONCLUSION}

This study is the first phenomenological study to describe clinical courage. Six features of clinical courage arose from conversations with rural doctors. These characteristics highlight the importance of family doctors' relationships with community and colleagues who are familiar with their contexts. Humility, preparation and the capacity to act when required enable family doctors to increase access to care for their patients. These attributes need to be developed and sustained in rural doctors.

\section{Author affiliations}

${ }^{1}$ Office of Rural and Regional Health, University of Alberta, Edmonton, Alberta, Canada

${ }^{2}$ Flinders Rural Health South Australia, Flinders University, Mount Gambier, South Australia, Australia

${ }^{3}$ Ukwanda Centre for Rural Health, Stellenbosch University, Stellenbosch, South Africa

${ }^{4}$ Rural Medicine, College or Medicine and Dentistry, James Cook University Faculty of Medicine Health and Molecular Sciences, Thursday Island, Queensland, Australia ${ }^{5}$ Australian College of Rural and Remote Medicine, Lakes Entrance, Victoria, Australia 
${ }^{6}$ Adelaide Rural Clinical School, The University of Adelaide Faculty of Health and Medical Sciences, Mount Gambier, South Australia, Australia

Twitter Jill Konkin @djillk1, Ruth Alison Stewart @RaatusRuth and Lucie Walters @ Lucie.WaltersRG

Contributors JK developed the study design and the interview questions, analysed and interpreted the data, and drafted the initial version of the manuscript. LG undertook the interviews, analysed the data, contributed to data interpretation and critical revision of the manuscript. EC undertook the interviews, analysed the data, contributed to data interpretation and critical revision of the manuscript. IC contributed to initial project design, data analysis and interpretation, and critical revision of the manuscript. RAS contributed to initial project design, data analysis and interpretation, and critical revision of the manuscript. DC contributed to initial project design, data analysis and interpretation, and critical revision of the manuscript. LW developed the study design, managed ethics application, developed the interview questions, analysed and interpreted the data, and drafted the manuscript. All approved the final manuscript.

Funding The authors have not declared a specific grant for this research from any funding agency in the public, commercial or not-for-profit sectors.

Competing interests JK, IC, RAS, DC and LW all work as rural doctors and have leadership roles in rural medical undergraduate or vocational training. LG and EC both spent a full academic year as medical students studying in rural sites. Australian Rural Clinical Schools are funded through the Australian Government grant scheme Rural Health Multidisciplinary Training.

Patient consent for publication Not required.

Ethics approval Ethics approval was obtained for the study through Flinders University Social and Behavioural Research Ethics Committee (project no. 7612).

Provenance and peer review Not commissioned; externally peer reviewed.

Data availability statement Data are available on reasonable request. Consent was not obtained from participants for interview transcripts to be used by third parties.

Open access This is an open access article distributed in accordance with the Creative Commons Attribution Non Commercial (CC BY-NC 4.0) license, which permits others to distribute, remix, adapt, build upon this work non-commercially, and license their derivative works on different terms, provided the original work is properly cited, appropriate credit is given, any changes made indicated, and the use is non-commercial. See: http://creativecommons.org/licenses/by-nc/4.0/.

ORCID iD

Lucie Walters http://orcid.org/0000-0002-1322-1895

\section{REFERENCES}

1 Fors M. Geographical narcissism in psychotherapy: countermapping urban assumptions about power, space, and time. Psychoanal Psychol 2018;35:446-53.

2 Brems C, Johnson ME, Warner TD, et al. Barriers to healthcare as reported by rural and urban interprofessional providers. J Interprof Care 2006;20:105-18.

3 Isaac V, Walters L, McLachlan CS. Association between selfefficacy, career interest and rural career intent in Australian medical students with rural clinical school experience. BMJ Open 2015;5:e009574.

4 Wootton J. President's message. Clinical courage. Can J Rural Med 2011;16:45-6.

5 Maclellan K. Clinical courage. Can J Rural Med 2011;16:107.

6 Dunlop P. Clinical courage. Can J Rural Med 2011;16:107.

7 Vagel M. Crafting phenomenological research. Walnut Creek: California Left Coast Press Inc, 2014.

8 Creswell JW. Qualitative inquiry and research design: choosing among five approaches. Sage, 2012.

9 Mann KV. Theoretical perspectives in medical education: past experience and future possibilities. Med Educ 2011;45:60-8.

10 Walters L, Prideaux D, Worley P, et al. Demonstrating the value of longitudinal integrated placements to general practice preceptors. Med Educ 2011;45:455-63.

11 Hamric AB, Arras JD, Mohrmann ME. Must we be courageous? Hastings Cent Rep 2015;45:33-40.

12 Feigin S, Owens G, Goodyear-Smith F. Theories of human altruism: a systematic review. J Psychiatry Brain Funct 2018;1:5.

13 Crowden $A$. Virtue ethics and rural professional healthcare roles. Rural Soc 2010;20:64-75.

14 Bonilauri Ferreira APR, Ferreira RF, Rajgor D, et al. Clinical reasoning in the real world is mediated by bounded rationality: implications for diagnostic clinical practice guidelines. PLoS One 2010;5:e10265.

15 Walters L, Laurence CO, Dollard J, et al. Exploring resilience in rural GP registrars -implications for training. BMC Med Educ 2015;15:110.

16 Mylopoulos M, Kulasegaram K, Woods NN. Developing the experts we need: fostering adaptive expertise through education. J Eval Clin Pract 2018;24:674-7.

17 Bohle Carbonell K, Stalmeijer RE, Könings KD, et al. How experts deal with novel situations: a review of adaptive expertise. Educ Res Rev 2014;12:14-29.

18 Gruppen LD. Humility and respect: core values in medical education. Med Educ 2014;48:53-8.

19 Smith J, Hays R. Is rural medicine a separate discipline? Aust J Rural Health 2004;12:67-72.

20 Jobes DA. Collaborating to prevent suicide: a clinical-research perspective. Suicide Life Threat Behav 2000;30:8-17. 